\title{
Overpowering the component problem
}

\author{
Matthew R Bennett and Jeff Hasty \\ Department of Bioengineering and Institute for Nonlinear Science, University of California San \\ Diego, La Jolla, California, USA.
}

The physicist John von Neumann famously said (as quoted by Enrico Fermi to Freeman Dyson) $)^{1}$, "With four parameters I can fit an elephant, and with five I can make him wiggle his trunk." By this, von Neumann meant that if a mathematical model has too many unknown parameters, it can be manipulated to fit almost anything, making the value of the modeling exercise questionable. In synthetic biology, as in physics, such model 'overdetermination' is crippling, and pro gress in designing large circuits will hinge on improving our ability to predict the output of networks based on their governing architecture 2 . In this issue, Ellis et al. ${ }^{3}$ describe an important advance toward this goal that relies on the creation and modeling of a large library of promoters with well-quantified inputs and outputs. Using these promoters, the authors created genetic circuits that reliably behaved as predicted by computational modeling.

Among the many challenges that face synthetic biologists, the 'component problem' - that is, the paucity of well-characterized component parts-is imminently solvable. For example, at the transcriptional level, the most common negative regulators are the Lac and Tet promoters. These workhorses of synthetic biology come 'as is' - there is only one version of each from which to choose. And if a network requires more than one repressible promoter, the designer has few other options. Despite this lack of components, synthetic biology has had some major successes ${ }^{4-6}$, and a large fraction of successful synthetic networks have been built using these two promoters and their associated transcription factors. However, this situation is slowly changing as various groups begin to create libraries of synthetic parts ${ }^{7}$.

A consequence of the component problem is an issue that might be called the 'impedance problem'. This problem can be defined as the inability to change the dynamic range or regulatory strength of a network component so that it will work with another component without redesigning the entire network. For example, in the situation depicted in Figure 1, the designed network requires a regulatable promoter to drive a downstream signaling protein. The first experimental realization of the network might use the $\mathrm{P}_{\text {Lac }}$ promoter and the gene encoding the inhibitory transcription factor (LacI) that binds $\mathrm{P}_{\text {Lac }}$ (Fig. 1). If, after testing, the $\mathrm{P}_{\mathrm{Lac}}$ promoter is found to be too weak or too noisy, the researcher must find an alternative transcription factor-promoter pair that will increase the signal or change the frequency spectrum of the stochastic fluctuations ${ }^{8}$.

Because of the small number of existing components, however, this may require a complete redesign of the entire network. For instance, replacing the repressible Lac promoter with the repressible Tet promoter requires changes to the intermediate gene and to both the upstream

(c) 2009 Nature America, Inc. All rights reserved.

hasty@bioeng.ucsd.edu .

Synthetic gene networks can be readily redesigned using new libraries of quantitatively characterized promoters coupled with predictive mathematical modeling. 
and downstream networks. The upstream network must be reconfigured to produce the transcription factor TetR instead of LacI, and the downstream network must now receive a new input, in this case Gal4p (Fig. 1a). This is necessary because the Tet repressor (TetR) and the Tet transactivator (tTA) have the same binding domain and therefore interfere with one another.

Fortunately, the work of Ellis $e t a l .^{3}$ solves the impedance problem by solving the component problem. Using established experimental techniques, the authors screened numerous promoter variants with randomized, non-essential sequences to create a library of Lac and Tet promoters with different levels of transcriptional activity. They then fit these experimental data to computational models, which enabled the promoters to be incorporated into synthetic networks in a quantitative, predictable manner. By creating a library of promoters that are functionally the same but quantitatively different, they allowed the precise tuning of promoter strength without the need for network redesign. Therefore, researchers are no longer confined by the inherent strength of the standard Lac or Tet promoters. Instead, they can choose from a large array of variants of these promoters, each with a well-quantified transcriptional strength (Fig. 1b). Rather than redesigning the entire network shown in Figure 1, they can simply replace the weak promoter with a stronger version.

Ellis et al. ${ }^{3}$ went on to show that simple transcriptional control systems can be tuned by promoter choice. They used Lac and Tet promoters from their library to create a negative feedforward loop and a co-repressive toggle switch. In both circuits, they were able to use mathematical modeling to correctly predict how the behaviors of the networks would change when the promoters were swapped for others in the library. They also showed that these promoters can be used to create timers that are programmable by component choice. Their co-repressive toggle switch is used to drive the induction of $F L O 1$, which is responsible for the initiation of yeast sedimentation. By changing the relative strengths of the two promoters, the timing of the event can be changed predictably.

Of course, this work is only one step toward true 'tunability' of synthetic gene networks. There are many factors that go into the proper control of gene activity, and many avenues through which regulation occurs 9,10 . For instance, it may be possible to engineer libraries of transcription factors that are qualitatively the same (that is, they target the same promoter) but that vary quantitatively. With such a transcription factor library, synthetic biologists would be able to choose the regulatory strength, Hill coefficient or binding affinity of the transcription factor before network construction begins. One can also imagine tunable posttranscriptional regulators, such as kinases or proteases of differing efficiencies. Previous tunable synthetic systems have often involved small-molecule inducers that must be present in precise concentrations in the growth medium. Libraries of interchangeable parts of differing performance provide a much-needed alternative for synthetic biologists.

\section{References}

1. Dyson F. Nature 2004;427:297. [PubMed: 14737148]

2. Hasty J, McMillen D, Collins JJ. Nature 2002;420:224-230. [PubMed: 12432407]

3. Ellis T, Wang X, Collins JJ. Nat. Biotechnol 2009;27:465-471. [PubMed: 19377462]

4. Sprinzak D, Elowitz MB. Nature 2005;438:443-448. [PubMed: 16306982]

5. Tigges M, Marquez-Lago TT, Stelling J, Fussenegger M. Nature 2009;457:309-312. [PubMed: 19148099]

6. Stricker J, et al. Nature 2008;456:516-519. [PubMed: 18971928]

7. Canton B, Labno A, Endy D. Nat. Biotechnol 2008;26:787-793. [PubMed: 18612302]

8. Austin DW, et al. Nature 2006;439:608-611. [PubMed: 16452980] 
9. Isaacs FJ, Dwyer DJ, Collins JJ. Nat. Biotechnol 2006;24:545-554. [PubMed: 16680139]

10. Andrianantoandro E, Basu S, Karig DK, Weiss R. Mol. Syst. Biol 2006;2:2006.0028. [PubMed: 16738572] 


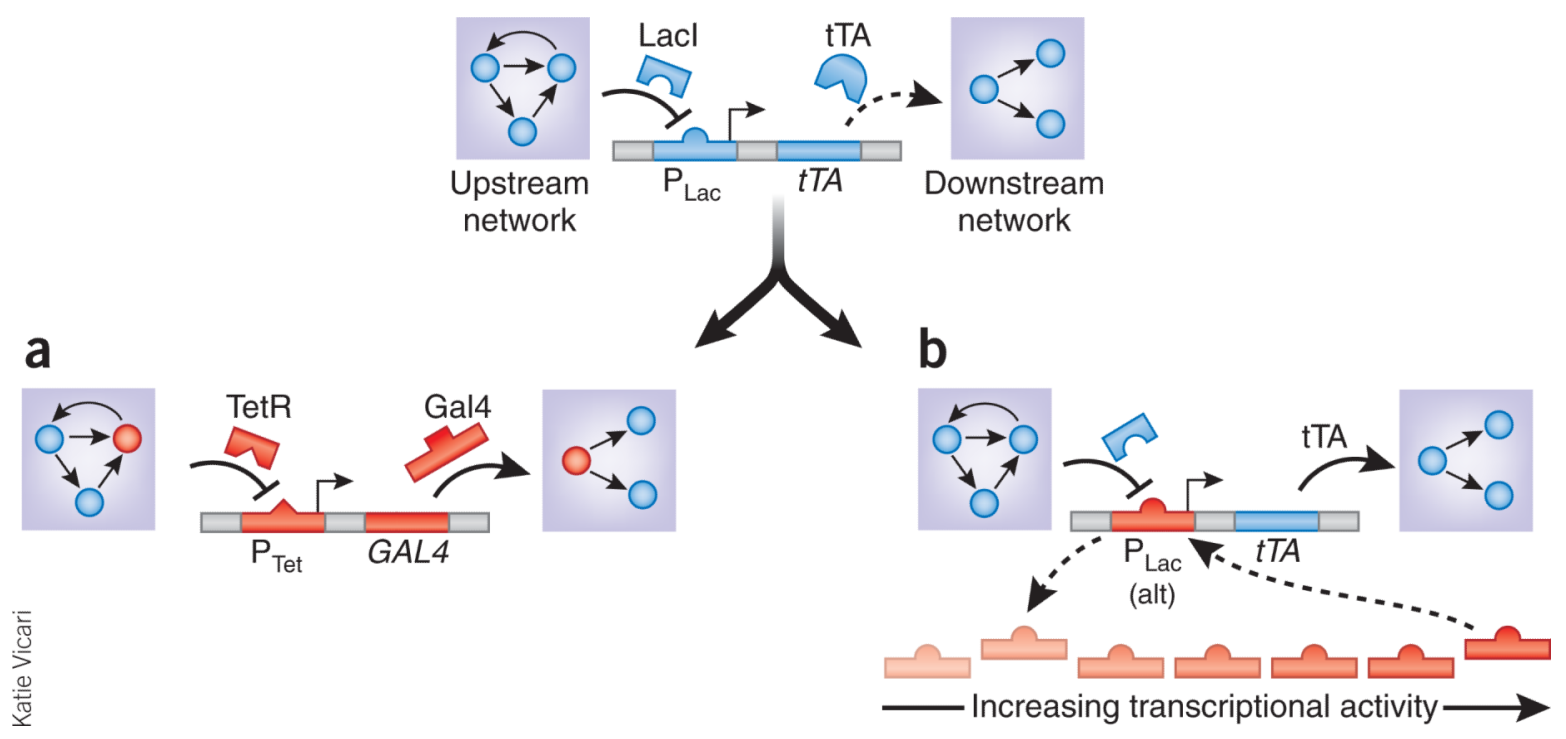

Figure 1.

Design and redesign of a synthetic gene network. In this example, the researcher wishes to create an intermediate signaling gene between upstream and downstream transcriptional networks. Initially, tTA is used to upregulate the downstream network. tTA is placed under the control of the $\mathrm{P}_{\text {Lac }}$ promoter, which is repressed by LacI produced upstream. However, the Lac promoter is too weak to produce the intended signal. (a) One possible solution is to replace the Lac promoter with a stronger promoter. Because of the lack of available transcription factor-promoter combinations, the best option is the repressible Tet promoter. This requires changes not just in the intermediate gene, but also in the upstream and downstream components (red indicates changed components). (b) With a library of Lac promoters of varying strengths, only one component must be changed to produce the desired output. 